\title{
Results of Mirogabalin Treatment for Diabetic Peripheral Neuropathic Pain in Asian Subjects: A Phase 2, Double-Blind, Randomized, Placebo-Controlled, Study
}

\author{
Masayuki Baba · Masanori Kuroha · Shoichi Ohwada · \\ Emiko Murayama · Norimitsu Matsui
}

Received: November 19, 2019 / Published online: February 12, 2020

(C) The Author(s) 2020

\section{ABSTRACT}

Introduction: Almost one-quarter of Asian patients with diabetes experience diabetic peripheral neuropathic pain (DPNP), which may be associated with moderate or severe levels of pain, insomnia, mood disorders, and worsened quality of life. Current treatments are generally ineffective and may be poorly tolerated. We evaluated mirogabalin as a treatment for DPNP in Asian subjects.

Methods: This phase 2, randomized, doubleblind, controlled study was conducted in Japan,

Enhanced Digital Features To view enhanced digital features for this article go to https://doi.org/10.6084/ m9.figshare.11698323.

Electronic supplementary material The online version of this article (https://doi.org/10.1007/s40122020-00156-6) contains supplementary material, which is available to authorized users.

M. Baba

Aomori Prefectural Central Hospital, Aomori, Japan

M. Kuroha $(\varangle) \cdot$ N. Matsui

Clinical Development Department, Daiichi Sankyo

Co., Ltd, Tokyo, Japan

e-mail: kuroha.masanori.kh@daiichisankyo.co.jp

S. Ohwada

Biostatistics and Data Management Department,

Daiichi Sankyo Co., Ltd, Tokyo, Japan

E. Murayama

Asia Development Department, Daiichi Sankyo Co., Ltd, Tokyo, Japan
South Korea, and Taiwan. Subjects $(n=450)$ with DPNP were randomized $(1: 1: 1: 1: 1)$ to treatment with 5,10 , or $15 \mathrm{mg}$ twice-daily (BID) mirogabalin, $150 \mathrm{mg}$ BID pregabalin, or placebo. The primary endpoint was change from baseline in average daily pain score (ADPS) at week 7; secondary endpoints included responder rates, Short-Form McGill Pain Questionnaire (SF-MPQ), Patient Global Impression of Change (PGIC), average daily sleep-interference score (ADSIS), and incidence of treatment-emergent adverse events (TEAEs).

Results: A greater improvement was noted for each mirogabalin treatment group for change from baseline in ADPS at week 7 compared with both placebo and with pregabalin, although these improvements were not statistically significant. The percentage of 30,50 , and $75 \%$ responders and subjects with PGIC improvements was greater in each mirogabalin group versus placebo. Mirogabalin $15 \mathrm{mg}$ BID significantly improved the SF-MPQ sensory $(p=0.0313)$ and visual analog scale scores $(p=0.0093)$, and ADSIS $(p=0.0002)$, versus placebo. Treatment was generally well tolerated; the most frequently reported TEAEs in the mirogabalin groups were somnolence $(14.7 \%)$ and dizziness (11.0\%), and most AEs were mild or moderate even at the highest dose.

Conclusions: In Asian subjects with DPNP, mirogabalin $(5,10$, and $15 \mathrm{mg}$ BID) was well tolerated. Although no significant differences were observed in the primary endpoint, there 
was a tendency toward improvement of pain with mirogabalin treatment, and this trend was also observed in the secondary endpoints.

Trial Registration: ClinicalTrials.gov identifier, NCT01504412.

Keywords: Clinical trial; Diabetic peripheral neuropathy; Mirogabalin; Pain management; Phase 2; Pregabalin

\section{Key Summary Points}

\section{Why carry out this study?}

Diabetic peripheral neuropathic pain (DPNP) is common in Asian patients with diabetes and may be associated with insomnia, mood disorders, and worsened quality of life, in addition to moderate or severe levels of pain.

Current treatments are generally ineffective, and may be poorly tolerated, resulting in compliance issues and discontinuation.

This study evaluated the hypothesis that at least one dosage level of mirogabalin would be effective and generally well tolerated as a treatment for DPNP in Asian subjects.

\section{What was learned from the study?}

Mirogabalin (5, 10, and $15 \mathrm{mg}$ BID) was well tolerated, and although no significant differences were observed in the primary endpoint, there was a tendency toward improvement of pain with mirogabalin treatment.

These data, combined with the positive outcomes from a US phase 2 proof-ofconcept study, suggest that further evaluation of mirogabalin in patients with DPNP would be worthwhile.

\section{INTRODUCTION}

In the past three decades, the number of people with diabetes has risen rapidly, with more than $60 \%$ of those affected living in Asia [1]. Up to $50 \%$ of patients with diabetes develop peripheral neuropathy [2], and a large proportion of those will experience diabetic peripheral neuropathic pain (DPNP) $[2,3]$. DPNP prevalence is reported to range from 4 to $22 \%$ in Asian patients with diabetes [4-6], although this may be lower than the true prevalence due to lack of awareness and under-reporting of the condition $[6,7]$. The symptoms of DPNP are commonly described as burning, shooting, tingling, and 'pins and needles', and most patients report moderate or severe levels of pain $[7,8]$. Individuals with DPNP have been reported to bear greater clinical and economic burdens compared with those with non-painful neuropathy $[7,9]$. DPNP is commonly associated with insomnia, mood disorders, and worsened quality of life (QOL) [3, 6], and has also been shown to be an independent predictor of depression [10].

The mechanisms underlying the development of DPNP are not well understood, which has resulted in a lack of effective treatment options for patients [2, 3]. Pregabalin and duloxetine are approved for the treatment of DPNP in the United States (US), and gabapentin is commonly used off-label [11-14]. However, these agents have limited efficacy and the high incidence of adverse events often results in treatment discontinuation [14, 15]. Data for other pharmacologic and non-pharmacologic therapies also suggest limited efficacy and safety concerns; studies are often confounded by poor methodology, and inconsistent endpoints, and rarely report QOL outcomes [16, 17].

Mirogabalin besylate (herein referred to as mirogabalin; Daiichi Sankyo Co., Ltd., Tokyo, Japan) was developed for the treatment of peripheral neuropathic pain, and was recently approved for this indication in Japan, based on the results of phase 3 studies [18-20]. Mirogabalin is an orally administered gabapentinoid that acts via $\alpha_{2} \delta$ calcium channel subunits to modulate pain transmission and processing 
[21]. It is thought that $\alpha_{2} \delta$ ligands act to relieve pain through the inhibition of abnormal neural transmissions, primarily in the posterior horn of the spinal cord $[22,23]$. In a rodent model of neuropathic pain that measured pain threshold, mirogabalin provided more potent effects than pregabalin, which is also an $\alpha_{2} \delta$ ligand [24]. The tolerability of mirogabalin has been confirmed in healthy Asian and Caucasian volunteers [25], and a phase 2 proof-of-concept study in US subjects with DPNP indicated that mirogabalin significantly reduced the average daily pain score (ADPS) compared with placebo [26].

The objective of this phase 2 study was to evaluate the hypothesis that at least one dosage level of mirogabalin would be effective and generally well tolerated as a treatment for DPNP in Asian subjects.

\section{METHODS}

\section{Study Design}

This was a phase 2, randomized, double-blind, placebo- and active comparator (pregabalin)controlled, parallel-group study (NCT01504412). The study was conducted in Japan (50 sites), South Korea (25 sites), and Taiwan (seven sites) from January 27, 2012 to June 20, 2013. The total study duration per subject was approximately 9 weeks, and consisted of an observation period (1 week), a treatment period (7 weeks), and a follow-up period after the last dose (1 week) (Fig. 1a).

Any subject who had been taking prohibited concomitant drugs underwent a washout period of 7 days or more, prior to the observation period. Prohibited medication requiring washout included pregabalin, antiepileptics (e.g., gabapentin, carbamazepine), antidepressants (except selective serotonin reuptake inhibitors [SSRIs]), hypnotics and anxiolytics (except for triazolam, zopiclone, zolpidem tartrate), opioids, tramadol, Neurotropin ${ }^{\circledR}, N$-methyl-D-aspartate receptor antagonists (e.g., dextromethorphan, ketamine, memantine), non-steroidal anti-inflammatory drugs, muscle relaxants, topical capsaicin, local anesthetics (e.g., lidocaine), sodium channel blockers (e.g., mexiletine), centrally acting sympatholytic agents (e.g., clonidine), steroids; peripheral vasodilators (e.g., cilostazol), Chinese herbal medicines with analgesic effects, vitamins B1 and B12, $\alpha$-lipoic acid, $\gamma$-linolenic acid (evening primrose oil), aldose reductase inhibitors, drugs that could cause irreversible retinal degeneration; and any other investigational product. Restricted medications, which had been administered at a stable dose for 30 days prior to screening and were continued during study treatment, included antidiabetic drugs (other than insulin), SSRI (only for depression and anxiety), hypnotics (triazolam, zopiclone, zolpidem tartrate only), vitamin B6 and other nutritional supplements, and aspirin (for prevention of thrombosis and embolism only). Prohibited non-pharmacologic therapies included nerve block, laser therapy, acupuncture; spinal cord stimulation, surgery, electrical stimulation therapy, and any other pain reduction therapy that might confound the study assessment.

\section{Ethics}

This study was conducted in compliance with the ethical principles that have their origin in the Declaration of Helsinki, the International Council for Harmonisation consolidated Guideline E6 for Good Clinical Practice (CPMP/ $\mathrm{ICH} / 135 / 95)$, and all applicable national and international regulatory requirements. The protocol and all associated documentation were approved by the Independent Ethics Committee or Institutional Review Board at each participating study center prior to study start (details of the individual IECs/IRBs are provided as Supplementary information). All subjects provided written informed consent prior to initiation of any study procedures.

\section{Subjects}

Subjects who met the following three criteria were considered to have DPNP and were further evaluated for study eligibility: bilateral neuropathic symptoms; etiology of peripheral neuropathy other than DPN was ruled out for these 
a

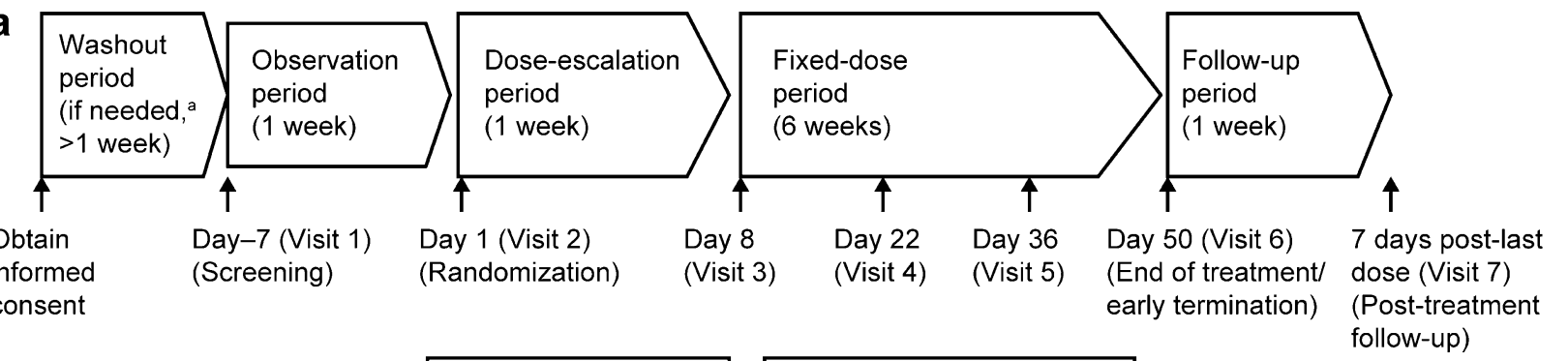

\begin{tabular}{|c|c|c|}
\hline Mirogabalin 5-mg BID group & Mirogabalin 5 mg QD & Mirogabalin 5 mg BID \\
\hline Mirogabalin 10-mg BID group & Mirogabalin $10 \mathrm{mg}$ QD & Mirogabalin $10 \mathrm{mg} \mathrm{BID}$ \\
\hline Mirogabalin 15-mg BID group & Mirogabalin $15 \mathrm{mg}$ QD & Mirogabalin $15 \mathrm{mg} \mathrm{BID}$ \\
\hline Placebo group & Placebo & Placebo \\
\hline Pregabalin group & Pregabalin 75 mg BID & Pregabalin 150 mg BID \\
\hline
\end{tabular}

b

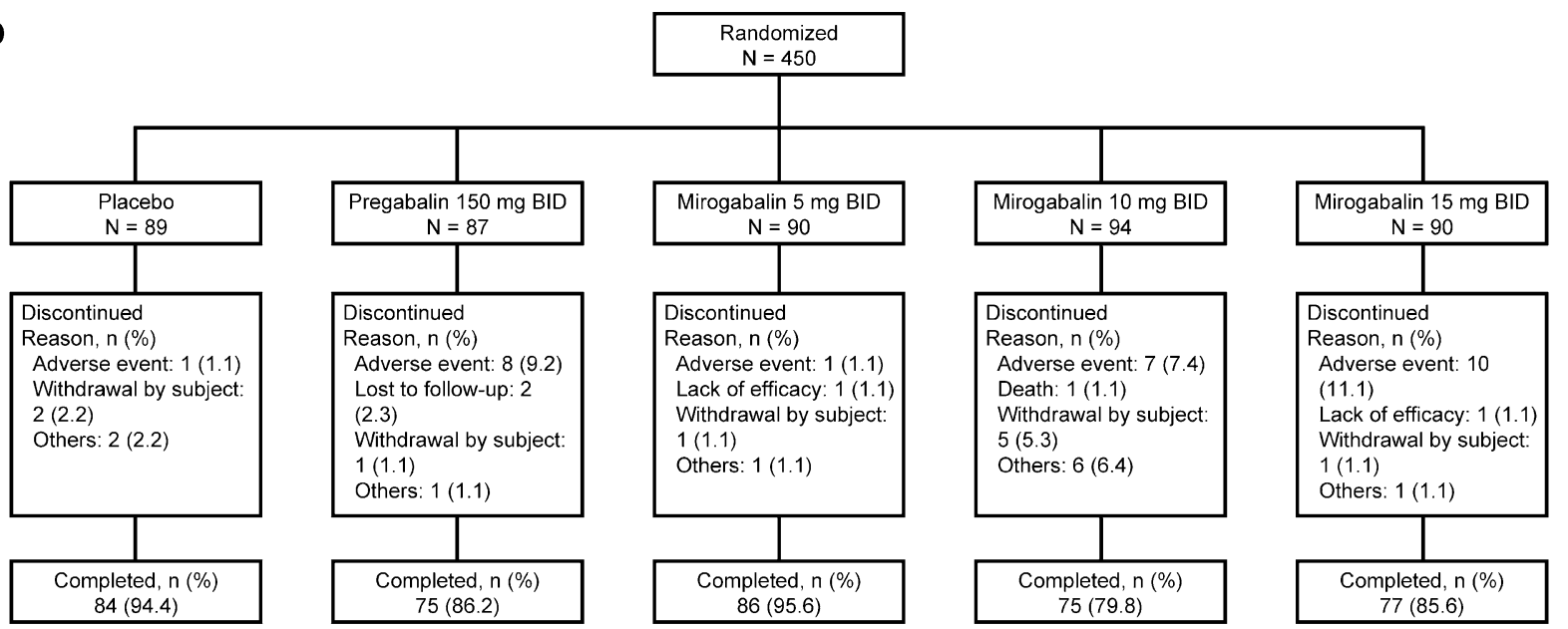

Fig. 1 Study design (a) and subject disposition (b). ${ }^{\mathrm{a}}$ After informed consent was obtained, subjects who were under treatment with the prohibited concomitant medications

symptoms; bilateral ankle jerk was decreased or absent. This DPNP assessment was conducted in accordance with the simplified diagnostic criteria for diabetic neuropathy established in Japan, and the diagnosis was made by a trained physician.

Evaluation of inclusion criteria and exclusion criteria was performed in the subjects who met the above-mentioned three conditions. Key inclusion criteria were age $\geq 20$ years; type 1 or type 2 diabetes mellitus at screening; painful distal symmetric polyneuropathy (diagnosed $\geq 6$ months prior to screening); a score underwent a washout period of 7 days or more. BID twice daily, $Q D$ once daily

of $\geq 40 \mathrm{~mm}$ on the Short-Form McGill Pain Questionnaire (SF-MPQ) visual analog scale (VAS) at both screening and randomization; and completion of at least four daily pain diaries with an ADPS numeric rating scale (NRS) score of $\geq 4$ over the past 7 days at the time of randomization. Key exclusion criteria were glycated hemoglobin $(\mathrm{HbA} 1 \mathrm{c})>9.0 \%$ at screening; uncontrolled blood glucose at screening or randomization that could require changes in diabetes treatment during the study period; any non-DPN-related severe pain or neurologic disorder, or skin conditions, that could confound 
the assessment of DPNP; amputation of body parts (except for toes) prior to screening or randomization; current or previous history of major psychiatric disorder, malignancy (except for basal cell carcinoma), pernicious anemia, untreated hypothyroidism, or any other clinically significant illness; creatinine clearance (using the Cockcroft-Gault equation) $<60 \mathrm{ml} /$ min at screening; hepatitis $\mathrm{B}$ or $\mathrm{C}$ or human immunodeficiency virus positive; previous treatment with pregabalin or gabapentin discontinued due to hypersensitivity, lack of efficacy, or intolerable adverse events (AEs); use of prohibited concomitant therapies within 7 days prior to screening or change of restricted concomitant drugs within 30 days prior to screening; drug or alcohol abuse within 12 months prior to screening; participation in another clinical study within 30 days of screening; suicidality (assessed using the Columbia-Suicide Severity Rating Scale [C-SSRS] [27]) at screening; pregnancy, lactation, or unwillingness to use contraception during the study and for 4 weeks after study completion; prior treatment with drugs that could cause irreversible retinal degeneration; and any other clinical or laboratory finding that rendered the subject ineligible at the discretion of the investigator.

\section{Treatment}

Eligible subjects were randomized using an interactive web response system in a 1:1:1:1:1 ratio to placebo, pregabalin $300 \mathrm{mg} /$ day, or one of three different doses of mirogabalin $(10,20$, or $30 \mathrm{mg} /$ day). Mirogabalin dose levels are expressed as the free form. Randomization was stratified by study site, so that the number of subjects per treatment group was approximately the same for each site.

For mirogabalin, a 1-week dose-escalation period at half of the fixed dose (administered at bedtime once daily) was implemented prior to the fixed-dose period. During the fixed-dose period, mirogabalin was administered orally twice daily ([BID] in the morning after breakfast and at bedtime) resulting in a 5-mg BID group, a 10-mg BID group, and a 15-mg BID group. For pregabalin, a 1-week dose-escalation period at a total daily dose of $150 \mathrm{mg}$ (75 mg BID), administered twice daily, was implemented prior to the fixed-dose period. During the fixed-dose period, pregabalin was administered at a dose of $150 \mathrm{mg}$ BID. The matching placebo was administered twice daily. Placebo was prepared to be indistinguishable in appearance from the investigational drug, with over-encapsulation used for both pregabalin and the pregabalin placebo.

\section{Study Endpoints}

The primary endpoint of this study was to compare mirogabalin with placebo based on change from baseline in ADPS at week 7, in subjects with DPNP.

Secondary endpoints were to characterize the dose-response effect of mirogabalin on the change from baseline in ADPS; to assess the incidence of responders, by treatment group, based on the proportion of subjects achieving $\mathrm{a} \geq 30 \%$ or $\geq 50 \%$ reduction from baseline in ADPS; to evaluate the effect of mirogabalin on pain using the SF-MPQ [28]: sensory, affective, and total subscales, VAS and present pain intensity) and the Patient Global Impression of Change (PGIC [29]); to assess the effect of mirogabalin on sleep using the average daily sleep-interference score (ADSIS); and to characterize the safety and tolerability of mirogabalin, based on the incidence of treatment-emergent adverse events (TEAEs; defined as any $\mathrm{AE}$ that appears for the first time during treatment, or that worsens relative to the pre-treatment state), discontinuations due to TEAEs, and changes in physical findings and results of safety monitoring.

\section{Measures}

ADPS and ADSIS were calculated based on entries in daily diaries, which were kept throughout the duration of the study. Every morning upon awakening, prior to taking study medication, the subject circled the number that best described his or her pain over the previous $24 \mathrm{~h}$ on a scale of 0 (no pain) to 10 (worst possible pain), and his or her sleep interference 
experience during the previous $24 \mathrm{~h}$ on a scale of 0 (pain did not interfere with sleep) to 10 (pain completely interfered with sleep). For ADPS, a minimally meaningful effect was defined as a mean decrease of at least 1.0 point (on a scale of 0 to 10 ) versus placebo.

The SF-MPQ was self-assessed at screening, randomization, at the end of dose-escalation (week 1), at weeks 3 and 5 (during the fixed dose period), and at the end of treatment (week 7). The SF-MPQ comprised 15 pain descriptors scored from 0 (none) to 3 (severe), which were summarized as sensory, affective, and total scores, a 100-mm VAS, and a present pain intensity index from $0-5$. PGIC evaluation took place at the end of treatment. The PGIC measured the perception of change since randomization using a scale of 1 (very much improved) to 7 (very much worse). AEs and physical and laboratory measurement values were recorded throughout the duration of the study. AEs were coded using the Medical Dictionary for Regulatory Activities (MedDRA).

\section{Statistical Analysis}

The planned sample size was approximately 400 subjects, with 80 subjects per treatment group, providing $83 \%$ statistical power to detect 1.1 units of change from baseline in ADPS between mirogabalin and placebo. The statistical power was based on analysis of variance (ANOVA) using a Dunnett-type adjustment for multiple comparison, a one-sided test with a significance level of 0.025 , and the assumption of a standard deviation for change from baseline in ADPS of 2.1 units in all treatment groups.

The full analysis set (FAS), defined as all randomized subjects who had received at least one dose of study medication and had baseline and at least one post-randomization pain rating, was used as the primary analysis set for all efficacy analyses. The safety analysis set included all subjects who had received at least one dose of study medication.

Baseline ADPS was defined as the average of up to 7 (at least 4) available pain scores in the last 7 days at or before randomization. Similarly, baseline ADSIS was defined as the average of the last 7 (at least 1) available sleep interference scores at or before randomization. For the other parameters, baseline value was defined as the last non-missing available value at or before randomization.

The last observation carried forward (LOCF) approach was used to impute missing ADPS at week 7. The change from baseline in ADPS at week 7 for each mirogabalin dosage group was compared with that for placebo, using an analysis of covariance (ANCOVA) model with treatment (including the pregabalin group) as a fixed effect and baseline ADPS as a covariate. Dunnett's method was used for the adjustment of multiple comparisons between each mirogabalin dose and placebo. The same ANCOVA model was used for the comparison between mirogabalin and pregabalin, and pregabalin and placebo, without multiple adjustment. The baseline observation carried forward (BOCF) approach was also used as a sensitivity analysis.

For all secondary endpoints, no adjustment was made for multiple comparisons. For the responder rates, and PGIC, a Chi square test without continuity correction (CC) was performed. The difference and the corresponding 95\% confidence intervals (CI) were estimated by week, using the Newcombe-Wilson score method without CC. The change from baseline in SF-MPQ at week 7 (LOCF imputation) was compared using the ANCOVA model with baseline covariate. The change from baseline in ADSIS at week 7 (LOCF imputation) for each mirogabalin group was compared with that for placebo, using the ANCOVA model with baseline ADSIS as the covariate.

All statistical analyses were performed using Statistical Analysis Software (SAS) version 9.2 (SAS Institute Inc., Cary, NC, USA).

\section{RESULTS}

\section{Subjects}

Overall, 736 subjects gave informed consent, and 450 were randomized to one of the five treatment groups (mirogabalin 5-mg BID, $n=90 ; \quad$ mirogabalin $10-\mathrm{mg}$ BID, $n=94$; mirogabalin $15-\mathrm{mg} \mathrm{BID}, n=90$; pregabalin, 
$n=87$; placebo, $n=89$ ). The study was completed by $86,75,77,75$, and 84 subjects, respectively. The most common reason for discontinuation was the occurrence of an $\mathrm{AE}$ (Fig. 1b).

A total of 446 subjects were included in the FAS (5-mg BID, $n=90 ; 10 \mathrm{mg}$ BID, $n=93$; 15-mg BID, $n=90$; pregabalin, $n=85$; placebo, $n=88$ ). Of the four subjects excluded from the FAS, three (one subject each in the placebo group, the pregabalin group, and the 10-mg BID group) were excluded because of major protocol violations, and one in the pregabalin group was excluded due to a lack of primary efficacy data (pain score) after randomization. The three subjects with protocol violations were also excluded from the safety analysis set, which consequently comprised 447 subjects.

Demographic and other baseline characteristics are reported in Table 1 . No notable differences were found between treatment groups. Overall, the mean age was 59.8 years, and approximately two-thirds of subjects $(64.6 \%)$ were male. The mean body weight was $67.7 \mathrm{~kg}$, and the mean creatinine clearance was $91.3 \mathrm{ml} /$ min. As expected, since type 2 diabetes mellitus is overwhelmingly more common in Asia than type 1 , almost all subjects (97.1\%) had type 2 diabetes, and the median duration of diabetes was 11.0 years. The median duration of DPN was 46.0 months and that of DPNP was 36.0 months.

\section{Efficacy Outcomes}

\section{Primary Endpoint}

The mean baseline ADPS was 5.92 for subjects in the FAS. The least squares mean (LSM) changes from baseline in ADPS at week 7 were -1.9 in the 5-mg BID group, -1.8 in the 10-mg BID group, -1.7 in the 15 -mg BID group, -1.4 in the pregabalin group, and -1.5 in the placebo group (Table 2, Fig. 2). There was a small, nonstatistically significant improvement for each mirogabalin group in LSM ADPS compared with placebo, although the improvements were not dose dependent. The LSM change in ADPS in the pregabalin group was similar to that in the placebo group.
The LSM placebo-adjusted difference (95\% CI) in change from baseline in ADPS at week 7 was $-0.4(-1.0$ to 0.2$)$ in the 5-mg BID group, -0.4 ( -0.9 to 0.2 ) in the 10-mg BID group, -0.3 ( -0.9 to 0.3 ) in the 15-mg BID group, and 0.0 ( -0.5 to 0.5$)$ in the pregabalin group. These differences versus placebo were not statistically significant for any treatment group (Table 2).

\section{Secondary and Exploratory Endpoints}

At week 7, the percentage of 30\% and 50\% responders was greater in each mirogabalin group compared with placebo, but these differences were not statistically significant (Table 2).

The LSM change from baseline in SF-MPQ total score at week 7 showed a numeric improvement in each mirogabalin group compared with the placebo group (Table 2). The placebo-adjusted LSM difference was statistically significant in the 15-mg BID group ( -1.9 , $95 \%$ CI -3.6 to $-0.2 ; p=0.0313)$, primarily driven by changes in the sensory score rather than the affective score. Similarly, the LSM change from in the VAS score was numerically greater in each mirogabalin group vs. placebo, with a statistically significant placebo-adjusted difference in the 15-mg BID group (-7.4, 95\% CI -13.0 to $-1.8 ; p=0.0093)$. No statistically significant differences between any of the mirogabalin groups and the placebo group were observed for the present pain intensity index, and no statistically significant differences in any SF-MPQ parameters were observed for pregabalin versus placebo.

At the end of treatment/early termination, the percentage of subjects with PGIC score of minimally improved or better (score $\leq 3$ ) was greater in each mirogabalin group than in the placebo group (Table 2). The placebo-adjusted difference was statistically significant in the 5-mg BID group (13.9\%, 95\% CI 0.9-26.3; $p=0.0356)$. The percentage of subjects with PGIC score of much improved or better (score $\leq 2$ ) was also greater in each mirogabalin group compared with placebo, but none of the differences were statistically significant.

The LSM change from baseline in ADSIS at week 7 was numerically greater in each mirogabalin group than in the placebo group 
Table 1 Baseline demographic and clinical characteristics (full analysis set)

\begin{tabular}{|c|c|c|c|c|c|c|}
\hline \multirow[t]{2}{*}{ Parameter } & \multicolumn{3}{|l|}{ Mirogabalin } & \multirow{2}{*}{$\begin{array}{l}\text { Pregabalin } \\
150 \mathrm{mg} \text { BID } \\
n=85\end{array}$} & \multirow{2}{*}{$\begin{array}{l}\text { Placebo } \\
n=88\end{array}$} & \multirow{2}{*}{$\begin{array}{l}\text { Total } \\
N=446\end{array}$} \\
\hline & $\begin{array}{l}5 \mathrm{mg} \text { BID } \\
n=90\end{array}$ & $\begin{array}{l}10 \mathrm{mg} \text { BID } \\
n=93\end{array}$ & $\begin{array}{l}15 \mathrm{mg} \text { BID } \\
n=90\end{array}$ & & & \\
\hline Age (years), mean (SD) & $60.5(9.8)$ & $60.7(9.3)$ & $59.0(10.1)$ & $60.2(8.8)$ & $58.4(9.9)$ & $59.8(9.6)$ \\
\hline \multicolumn{7}{|l|}{ Gender } \\
\hline Male & $59(65.6)$ & $58(62.4)$ & $49(54.4)$ & $60(70.6)$ & $62(70.5)$ & $288(64.6)$ \\
\hline Female & $31(34.4)$ & $35(37.6)$ & $41(45.6)$ & $25(29.4)$ & $26(29.5)$ & $158(35.4)$ \\
\hline Weight $(\mathrm{kg})$, mean $(\mathrm{SD})$ & $68.0(10.1)$ & $66.1(10.8)$ & $67.0(14.8)$ & $69.0(12.5)$ & $68.4(13.2)$ & $67.7(12.4)$ \\
\hline $\begin{array}{l}\text { Creatinine clearance }^{\mathrm{a}}(\mathrm{ml} / \\
\text { min), mean }(\mathrm{SD})\end{array}$ & $91.3(31.2)$ & $90.2(27.9)$ & $92.4(31.7)$ & $91.6(30.0)$ & $91.3(23.5)$ & $91.3(28.9)$ \\
\hline Baseline $\mathrm{ADPS}^{\mathrm{b}}$, mean (SD) & $5.8(1.2)$ & $5.9(1.4)$ & $5.9(1.3)$ & $6.1(1.5)$ & $6.0(1.2)$ & $5.9(1.3)$ \\
\hline \multicolumn{7}{|l|}{ Type of diabetes mellitus } \\
\hline Type 1 & $1(1.1)$ & $3(3.2)$ & $5(5.6)$ & $1(1.2)$ & $3(3.4)$ & $13(2.9)$ \\
\hline Type 2 & $89(98.9)$ & $90(96.8)$ & $85(94.4)$ & $84(98.8)$ & $85(96.6)$ & $433(97.1)$ \\
\hline $\begin{array}{l}\text { Duration of diabetes (years), } \\
\text { median (range) }\end{array}$ & $10.0(0,39)$ & $13.0(0,33)$ & $10.0(0,38)$ & $12.0(0,40)$ & $10.0(1,40)$ & $11.0(0,40)$ \\
\hline $\begin{array}{l}\text { Duration of DPN (months), } \\
\text { median (range) }\end{array}$ & $48.0(6,221)$ & $60.0(6,332)$ & $41.5(6,411)$ & $43.0(6,233)$ & $34.0(6,209)$ & $46.0(6,411)$ \\
\hline $\begin{array}{l}\text { Duration of DPNP } \\
\text { (months), median (range) }\end{array}$ & $36.0(6,165)$ & $41.0(6,332)$ & $36.0(6,411)$ & $32.0(6,152)$ & $32.0(6,209)$ & $36.0(6,411)$ \\
\hline
\end{tabular}

Data are given as $n(\%)$ unless otherwise stated

$A D P S$ average daily pain score, $B I D$ twice daily, $D P N P$ diabetic peripheral neuropathic pain, $S D$ standard deviation

a The creatinine clearance was calculated based on the Cockcroft-Gault equation using age, values of body weight and serum creatinine at screening

b The baseline ADPS was defined as the average of up to seven available pain scores in the last 7 days at or before randomization

(Table 2). The placebo-adjusted LSM difference was statistically significant in the $15-\mathrm{mg}$ BID group $(-0.9,95 \%$ CI -1.3 to -0.4 ; $p=0.0002)$.

\section{Safety Outcomes}

The median treatment duration was 50.0 days for all treatment groups, with no notable differences among groups. The incidence of TEAEs was generally similar across treatment groups. In the mirogabalin groups, TEAEs increased slightly with dose, from $48.9 \%$ for the 5-mg BID group, $53.4 \%$ for the $10-\mathrm{mg}$ BID group, to $73.3 \%$ for the 15-mg BID group. The corresponding TEAE rates for pregabalin and placebo were 58.1 and $53.4 \%$, respectively. The incidence of serious TEAEs was $<5 \%$ and similar in all treatment groups. No serious TEAEs were considered related to study treatment by the investigator. Two deaths (one each in the mirogabalin 5-mg and 10-mg BID groups) due to completed suicide were reported; both events were considered by the investigator to be unrelated to the study drug. 


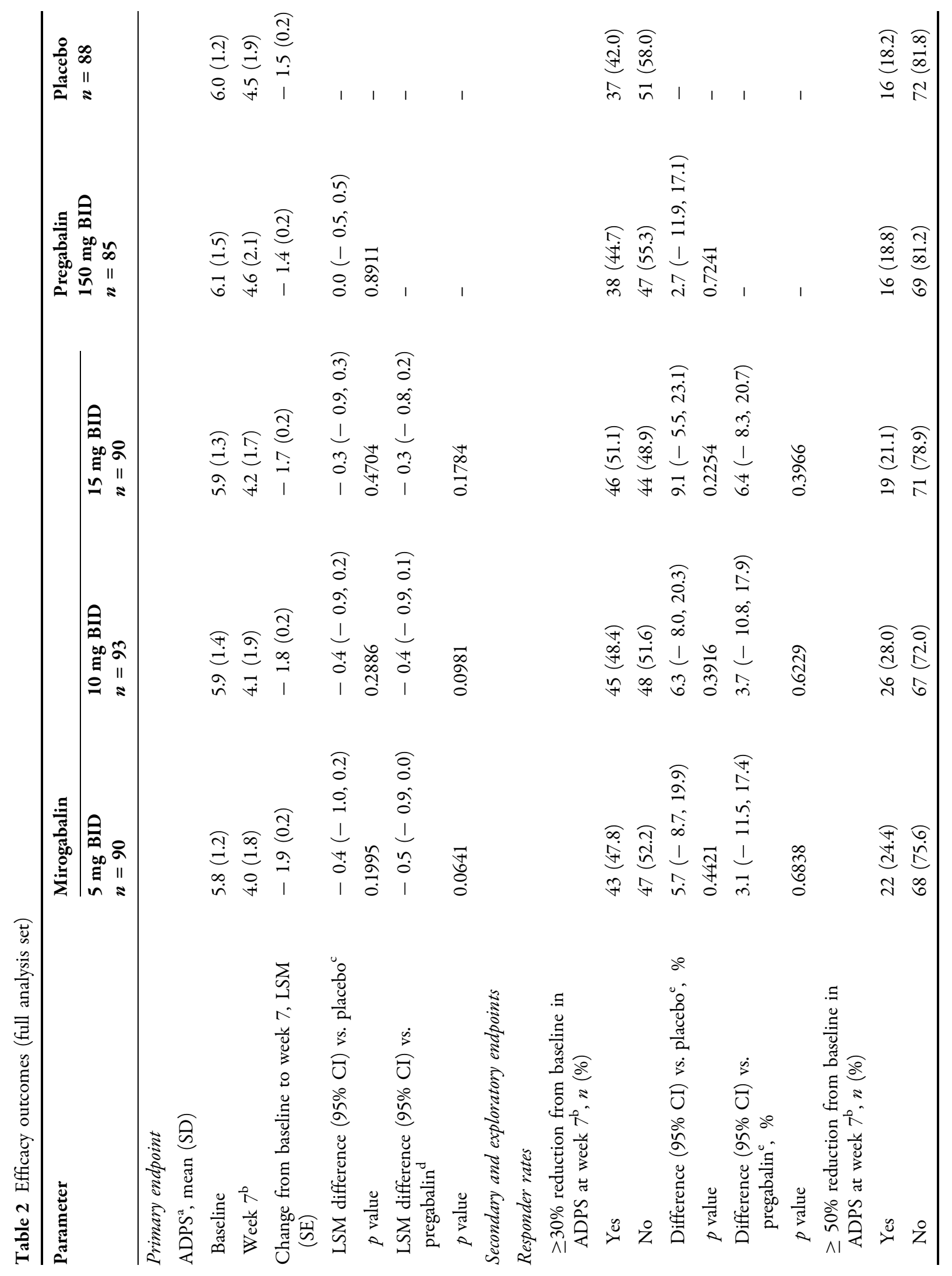




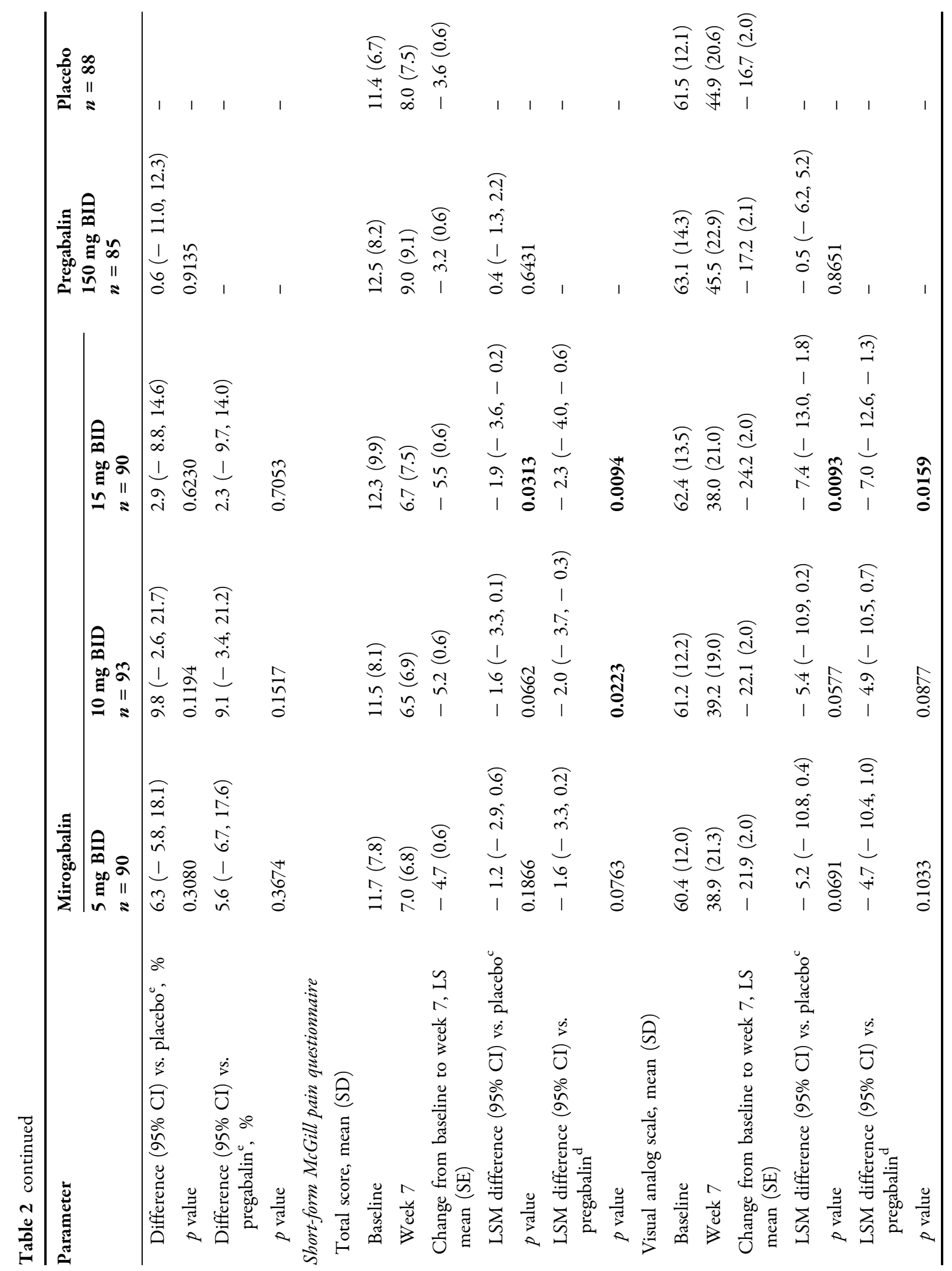




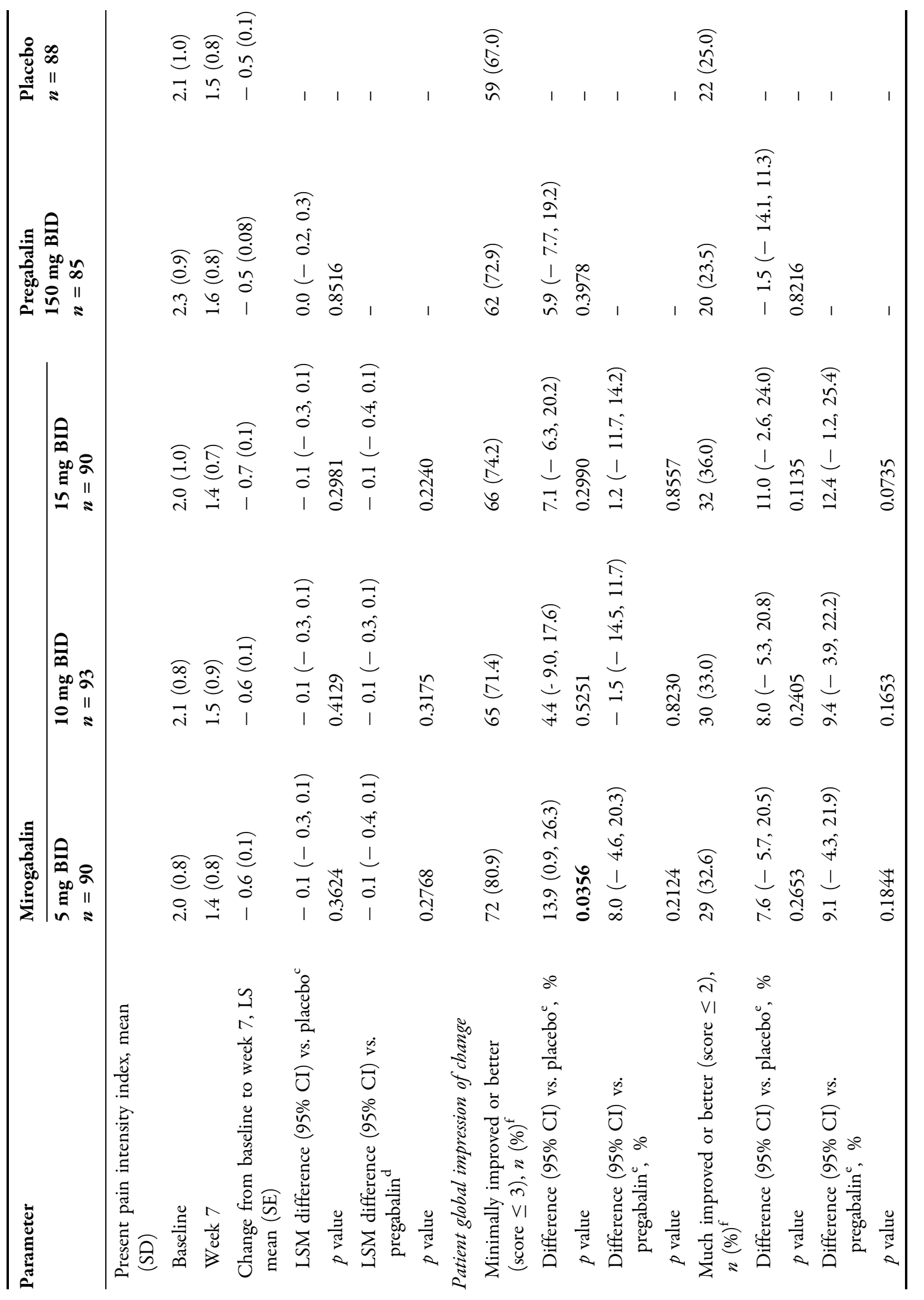




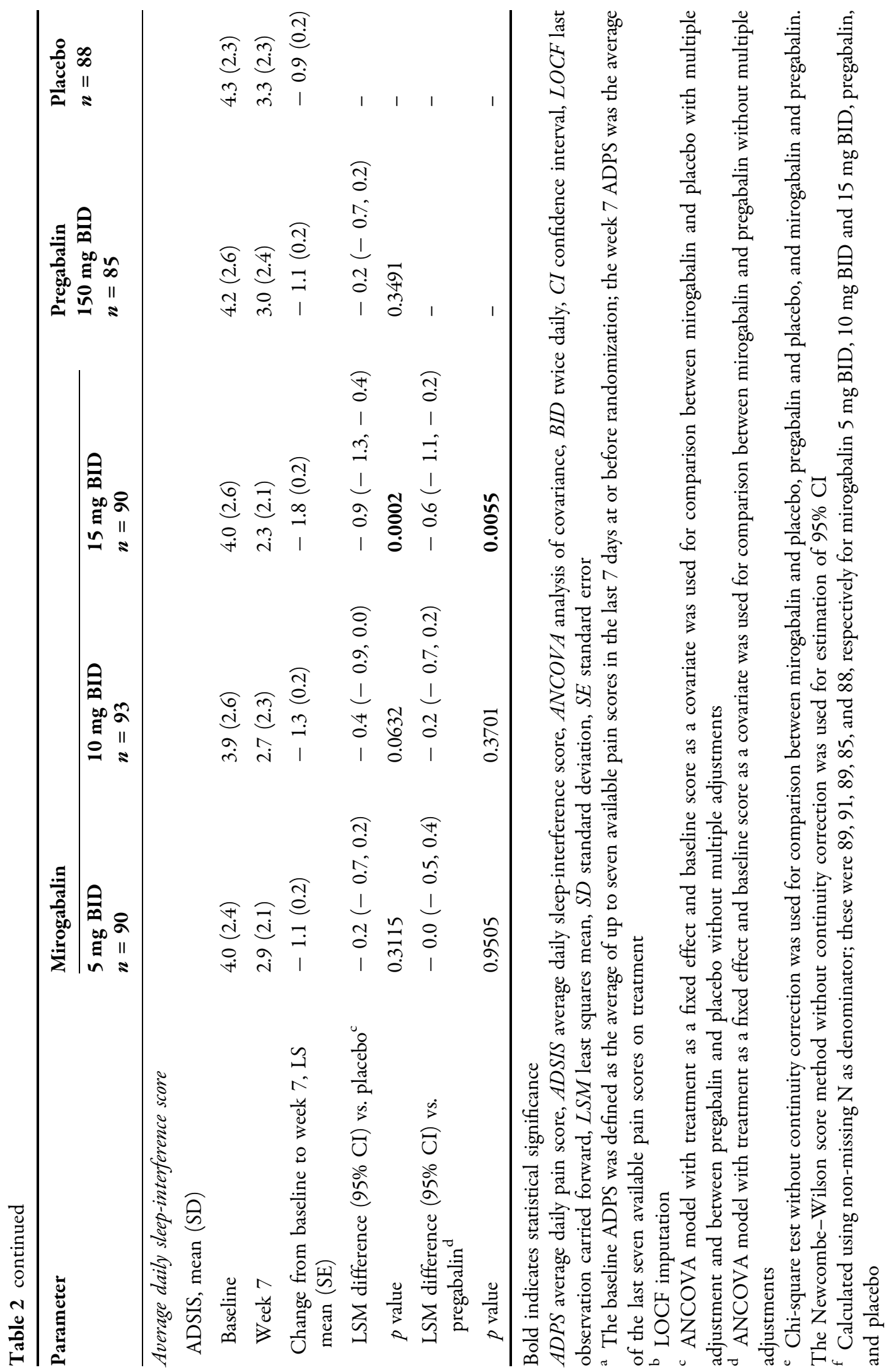




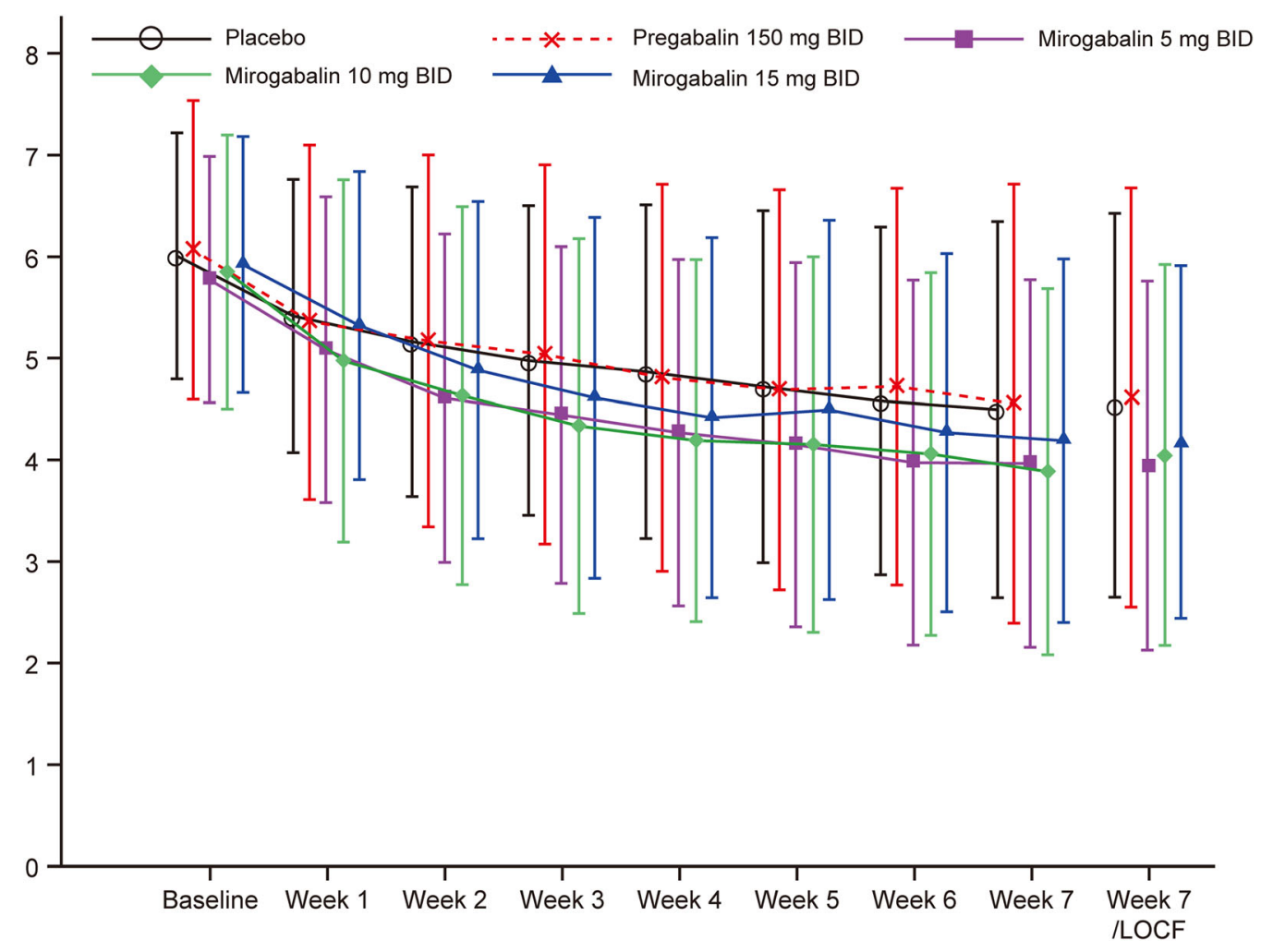

Fig. 2 Time course of average daily pain scores ${ }^{a}$ (full analysis set). ADPS average daily pain score, $B I D$ twice daily, $L O C F$ last observation carried forward, $S D$ standard

The incidence of TEAEs leading to treatment discontinuation was $7.3 \%$ in the mirogabalin groups combined, $8.1 \%$ in the pregabalin group, and $1.1 \%$ in the placebo group. In the mirogabalin groups, discontinuation rates increased as the dosage increased. Across all mirogabalin treatment groups, the majority of TEAEs experienced were mild or moderate in severity. No clear dose-related trends in the incidence of severe TEAEs were found in the mirogabalin groups.

The most frequently reported TEAEs in the mirogabalin groups were somnolence $(14.7 \%)$, dizziness $(11.0 \%)$, and nasopharyngitis $(8.4 \%)$ (Table 3). As the dose of mirogabalin increased, the incidence of somnolence and dizziness increased. The only other TEAEs occurring with an incidence of greater than $5 \%$ in any mirogabalin treatment group were edema peripheral in the $15-\mathrm{mg}$ BID group $(7.8 \%)$, gait disturbance in the 10-mg BID group (7.5\%), and deviation. ${ }^{a}$ Arithmetic mean (SD). The baseline ADPS was defined as the average of up to seven available pain scores in the last 7 days at or before randomization

weight increase in the 15-mg BID group (5.6\%). For the placebo group, the most common TEAE was somnolence $(5.7 \%)$. For the pregabalin group, the most common TEAEs were somnolence $(14.0 \%)$ and dizziness (10.5\%). No clinically significant alterations in clinical physical or laboratory test results were reported, with the exception of one subject in the $150 \mathrm{mg}$ BID group who experienced a clinically significant abnormality in 12-lead electrocardiogram (mild transient atrial fibrillation at the end of study treatment which resolved 1 day later).

\section{DISCUSSION}

Although it is widely known that patients with DPNP report considerable levels of pain [8], worsened QOL [3], and comorbidities including depression [10] and insomnia [6], to date, effective treatments are lacking. Mirogabalin, 
Table 3 Summary of the most frequently reported treatment-emergent adverse events (at least 5\% of subjects in any treatment group) by SOC and PT (safety analysis set)

\begin{tabular}{|c|c|c|c|c|c|c|}
\hline & \multicolumn{4}{|c|}{ Mirogabalin } & \multirow{2}{*}{$\begin{array}{l}\text { Pregabalin } \\
150 \mathrm{mg} \text { BID } \\
n=85\end{array}$} & \multirow{2}{*}{$\begin{array}{l}\text { Placebo } \\
n=88\end{array}$} \\
\hline & $\begin{array}{l}5 \mathrm{mg} \text { BID } \\
n=90\end{array}$ & $\begin{array}{l}10 \mathrm{mg} \text { BID } \\
n=93\end{array}$ & $\begin{array}{l}15 \mathrm{mg} \text { BID } \\
n=90\end{array}$ & $\begin{array}{l}\text { All dosages } \\
n=273\end{array}$ & & \\
\hline \multicolumn{7}{|c|}{ Infections and infestations } \\
\hline Nasopharyngitis & $7(7.8)$ & $13(14.0)$ & $3(3.3)$ & $23(8.4)$ & $2(2.3)$ & $3(3.4)$ \\
\hline \multicolumn{7}{|c|}{ Nervous system disorders } \\
\hline Somnolence & $8(8.9)$ & $13(14.0)$ & $19(21.1)$ & $40(14.7)$ & $12(14.0)$ & $5(5.7)$ \\
\hline Dizziness & $5(5.6)$ & $10(10.8)$ & $15(16.7)$ & $30(11.0)$ & $9(10.5)$ & $3(3.4)$ \\
\hline Headache & $2(2.2)$ & $4(4.3)$ & $3(3.3)$ & $9(3.3)$ & $5(5.8)$ & $2(2.3)$ \\
\hline \multicolumn{7}{|c|}{ Gastrointestinal disorders } \\
\hline Vomiting & $1(1.1)$ & $3(3.2)$ & $2(2.2)$ & $6(2.2)$ & $5(5.8)$ & $1(1.1)$ \\
\hline \multicolumn{7}{|c|}{ General disorders and administration site conditions } \\
\hline Edema peripheral & $3(3.3)$ & $3(3.2)$ & $7(7.8)$ & $13(4.8)$ & $5(5.8)$ & $1(1.1)$ \\
\hline Gait disturbance & $1(1.1)$ & $7(7.5)$ & $2(2.2)$ & $10(3.7)$ & $4(4.7)$ & $1(1.1)$ \\
\hline \multicolumn{7}{|l|}{ Investigations } \\
\hline Weight increased & $1(1.1)$ & $2(2.2)$ & $5(5.6)$ & $8(2.9)$ & $2(2.3)$ & $0(0.0)$ \\
\hline
\end{tabular}

Data are given as $n(\%)$

Adverse events were coded using the Medical Dictionary for Regulatory Activities version 16.0

$B I D$ twice daily, $P T$ preferred term, SOC system organ class

an orally administered gabapentinoid, was developed as a new therapeutic agent for the treatment of peripheral neuropathic pain. This Asian, phase 2, multicenter, randomized, double-blind, placebo- and pregabalin-controlled, parallel-group study was conducted to evaluate the efficacy of mirogabalin at doses of $5 \mathrm{mg}$ BID, $10 \mathrm{mg}$ BID, and $15 \mathrm{mg}$ BID in subjects with DPNP.

The primary endpoint of this study was change from baseline in ADPS at week 7. A numerically greater improvement was noted for each of the three mirogabalin treatment groups compared with both placebo and with pregabalin, although these improvements were not statistically significant. With regard to the secondary efficacy endpoints in this study, mirogabalin improved the sensory score and VAS score of SF-MPQ, and improved ADSIS compared with placebo, with statistically significant differences observed in the mirogabalin 15-mg BID group. Overall, these data suggested that mirogabalin could provide painrelated benefit for patients.

The design of clinical trials to evaluate treatments for neuropathic pain has been problematic, with inconsistent efficacy results and high placebo responses [30-32]. In order to appropriately assess the efficacy of the study drug, this study was limited to subjects who experienced pain of moderate or greater intensity, which would also be aligned with the pain intensities reported in the general DPNP population [8]. Pregabalin was selected as the active control since both agents target the same voltage-gated calcium channel $\alpha_{2} \delta$ subunit, and because pregabalin is approved in many countries for the treatment of DPNP [13, 15]. No upper age limit was placed on this study, since a phase 1 study in elderly subjects showed no 
notable differences from the results obtained in non-elderly subjects with regard to safety, tolerability, and pharmacokinetics [33]. Nevertheless, despite these elements of study design, mirogabalin produced numeric but not statistically significant improvements compared with placebo, and almost no impact was observed with pregabalin on any measure, likely due to the subjective nature of pain measurement and the difficulty in minimizing the placebo response [34]. Moreover, it must be noted that the baseline ADPS across all treatment groups in this study were lower compared with the baseline pain values in the US phase 2 proof-ofconcept study [26], which may have limited the ability to observe meaningful reductions in ADPS.

In general, mirogabalin was well tolerated in this study. In all treatment groups, somnolence was the most frequently reported TEAE $(14.7 \%$ across mirogabalin groups, $5.7 \%$ in the placebo group, and $14.0 \%$ in the pregabalin group). In comparison, the most common TEAEs in the US phase 2 proof-of-concept study were dizziness $(7.6 \%)$ and somnolence $(5.1 \%)$ across all mirogabalin groups, and somnolence $(8.0 \%)$ in the pregabalin $300 \mathrm{mg} /$ day group [26]. While the rates of somnolence and other TEAEs in the Japanese subjects in our study were slightly higher than in the US study, they were noticeably lower than those previously reported to be associated with pregabalin treatment; in a 14-week placebo-controlled trial of pregabalin (300 or $600 \mathrm{mg} /$ day) for DPNP, somnolence (26\%), dizziness (24\%), and peripheral edema (13\%) were the most frequently reported AEs [35].

Here, as the dose of mirogabalin increased, the incidence of TEAEs increased, and the incidence of TEAEs related to dizziness, somnolence, and edema also increased. However, the incidence of severe TEAEs did not increase as the dose of mirogabalin increased, and most TEAEs were mild or moderate even at the highest dose (15 mg BID). Furthermore, the incidence of serious TEAEs did not increase as the dose of mirogabalin increased. Of note, no subject in this study experienced any abnormalities in laboratory parameters suggestive of drug-induced liver injury, despite the fact that a potential Hy's law case was reported in a US phase 2 study of mirogabalin for DPNP [26]. Two deaths due to completed suicide were reported in subjects receiving mirogabalin. Although a causal relationship between these suicides and mirogabalin was not established (in the opinion of the investigator), this may need to be monitored in future studies.

We acknowledge that this study has some limitations, which should be considered when evaluating the data. These include the short study duration (7 weeks), which precludes the collection of long-term safety data, which are needed to affirm the safety profile of any pharmacologic agent intended to treat a chronic condition. In addition, the restriction of study participants to those of Asian ethnicity may impede extrapolation of the resulting data to DPNP patients in other countries. Finally, patients in this study were required to have creatinine clearance $\geq 60 \mathrm{ml} / \mathrm{min}$, but there was no stratification based on renal function, which may have impacted drug exposure and response levels.

\section{CONCLUSIONS}

In subjects with DPNP receiving mirogabalin at dosages of $5 \mathrm{mg}$ BID, $10 \mathrm{mg}$ BID, or $15 \mathrm{mg}$ BID for 7 weeks including 1-week escalation period, treatment was well tolerated. Although no significant differences were observed in the primary endpoint, there was a tendency toward improvement of pain with mirogabalin treatment, and this trend was also observed in the secondary endpoints, suggesting that further evaluation of mirogabalin in this indication would be worthwhile.

\section{ACKNOWLEDGEMENTS}

Funding. This study was sponsored by Daiichi-Sankyo Co., Ltd., Tokyo, Japan. The sponsor also provided funding for the Rapid Service Fee. All authors had full access to all of the data in this study and take complete responsibility 
for the integrity of the data and accuracy of the data analysis.

Editorial Assistance. The authors wish to acknowledge editorial assistance provided by Sally-Anne Mitchell, PhD (McCANN HEALTH CMC, Japan), funded by Daiichi-Sankyo, Inc.

Authorship. All named authors meet the International Committee of Medical Journal Editors (ICMJE) criteria for authorship for this article, take responsibility for the integrity of the work as a whole, and have given their approval for this version to be published.

Disclosures. Masayuki Baba has received consultancy fees and speaker fees from Daiichi Sankyo Co., Ltd. Norimitsu Matsui, Masanori Kuroha, Emiko Murayama, and Shoichi Ohwada are employees of Daiichi Sankyo Co., Ltd.

Compliance with Ethics Guidelines. This study was conducted in compliance the ethical principles that have their origin in the Declaration of Helsinki, the International Council for Harmonisation consolidated Guideline E6 for Good Clinical Practice (CPMP/ICH/135/95), and all applicable national and international regulatory requirements. The protocol (DS5565A-J202) and all associated documentation were approved by the Independent Ethics Committee or Institutional Review Board (IEC/IRB) at each participating study center prior to study start (details of the individual IECs/IRBs are provided as Supplementary information). All subjects provided written informed consent prior to initiation of any study procedures.

Data Availability. The datasets generated during and/or analyzed during the current study are available from the corresponding author on reasonable request.

Open Access. This article is licensed under a Creative Commons Attribution-NonCommercial 4.0 International License, which permits any non-commercial use, sharing, adaptation, distribution and reproduction in any medium or format, as long as you give appropriate credit to the original author(s) and the source, provide a link to the Creative Commons licence, and indicate if changes were made. The images or other third party material in this article are included in the article's Creative Commons licence, unless indicated otherwise in a credit line to the material. If material is not included in the article's Creative Commons licence and your intended use is not permitted by statutory regulation or exceeds the permitted use, you will need to obtain permission directly from the copyright holder. To view a copy of this licence, visit http://creativecommons.org/licenses/by$\mathrm{nc} / 4.0 /$.

\section{REFERENCES}

1. Nanditha A, Ma RC, Ramachandran A, et al. Diabetes in Asia and the Pacific: implications for the global epidemic. Diabetes Care. 2016;39(3):472-85.

2. Sloan G, Shillo P, Selvarajah D, et al. A new look at painful diabetic neuropathy. Diabetes Res Clin Pract. 2018;144:177-91.

3. Shillo P, Sloan G, Greig M, et al. Painful and painless diabetic neuropathies: what is the difference? Curr Diabetes Rep. 2019;19(6):32.

4. Kim SS, Won JC, Kwon HS, et al. Prevalence and clinical implications of painful diabetic peripheral neuropathy in type 2 diabetes: results from a nationwide hospital-based study of diabetic neuropathy in Korea. Diabetes Res Clin Pract. 2014;103(3):522-9.

5. Pai YW, Lin CH, Lee IT, Chang MH. Prevalence and biochemical risk factors of diabetic peripheral neuropathy with or without neuropathic pain in Taiwanese adults with type 2 diabetes mellitus. Diabetes Metab Syndr. 2018;12(2):111-6.

6. Tsuji M, Yasuda T, Kaneto H, et al. Painful diabetic neuropathy in Japanese diabetic patients is common but underrecognized. Pain Res Treat. 2013;2013:318352.

7. Malik RA, Aldinc E, Chan SP, et al. Perceptions of painful diabetic peripheral neuropathy in SouthEast Asia: results from patient and physician surveys. Adv Ther. 2017;34(6):1426-37.

8. Paisley $P$, Serpell M. Improving pain control in diabetic neuropathy. Practitioner. 1802;2017(261): 23-6. 
9. Ebata-Kogure N, Nozawa K, Murakami A, et al. Clinical and economic burdens experienced by patients with painful diabetic peripheral neuropathy: an observational study using a Japanese claims database. PLoS One. 2017;12(10):e0187250.

10. D'Amato C, Morganti R, Greco C, et al. Diabetic peripheral neuropathic pain is a stronger predictor of depression than other diabetic complications and comorbidities. Diabetes Vasc Dis Res. 2016;13(6):418-28.

11. Iqbal Z, Azmi S, Yadav R, et al. Diabetic peripheral neuropathy: epidemiology, diagnosis, and pharmacotherapy. Clin Ther. 2018;40(6):828-49.

12. Eli Lilly and Company I, IN, USA. Cymbalta (duloxetine) prescribing information. https://pi.lilly. com/us/cymbalta-pi.pdf; 2017.

13. Pfizer Inc. NY, NY, USA. Lyrica (pregabalin) prescribing information. http://labeling.pfizer.com/ showlabeling. aspx?id=561; 2019.

14. Yang M, Qian C, Liu Y. Suboptimal treatment of diabetic peripheral neuropathic pain in the United States. Pain Med. 2015;16(11):2075-83.

15. Azmi S, ElHadd KT, Nelson A, et al. Pregabalin in the management of painful diabetic neuropathy: a narrative review. Diabetes Ther. 2019;10(1):35-56.

16. Waldfogel JM, Nesbit SA, Dy SM, et al. Pharmacotherapy for diabetic peripheral neuropathy pain and quality of life: a systematic review. Neurology. 2017;88(20):1958-67.

17. Amato Nesbit S, Sharma R, Waldfogel JM, et al. Non-pharmacologic treatments for symptoms of diabetic peripheral neuropathy: a systematic review. Curr Med Res Opin. 2018;17:1-11.

18. Deeks ED. Mirogabalin: first global approval. Drugs. 2019;79(4):463-8.

19. Kato J, Matsui N, Kakehi Y, et al. Mirogabalin for the management of postherpetic neuralgia: a randomized, double-blind, placebo-controlled phase 3 study in Asian patients. Pain. 2019;160(5):1175-85.

20. Baba M, Matsui N, Kuroha M, Wasaki Y, Ohwada S. Mirogabalin for the treatment of diabetic peripheral neuropathic pain: a randomized, double-blind, placebo-controlled phase III study in Asian patients. J Diabetes Investig. 2019;10:1299-306.

21. Domon $\mathrm{Y}$, Arakawa $\mathrm{N}$, Inoue $\mathrm{T}$, et al. Binding characteristics and analgesic effects of mirogabalin, a novel ligand for the alpha2delta subunit of voltage-gated calcium channels. J Pharmacol Exp Ther. 2018;365(3):573-82.
22. Field MJ, Li Z, Schwarz JB. Ca2 + channel alpha2delta ligands for the treatment of neuropathic pain. J Med Chem. 2007;50(11):2569-75.

23. Li CY, Zhang XL, Matthews EA, et al. Calcium channel alpha2delta1 subunit mediates spinal hyperexcitability in pain modulation. Pain. 2006;125(1-2):20-34.

24. Domon Y, Kitano Y, Makino M. Analgesic effects of the novel alpha(2)delta ligand mirogabalin in a rat model of spinal cord injury. Pharmazie. 2018;73(11):659-61.

25. Jansen $\mathrm{M}$, Warrington $\mathrm{S}$, Dishy $\mathrm{V}$, et al. A randomized, placebo-controlled, double-blind study of the safety, tolerability, pharmacokinetics, and pharmacodynamics of single and repeated doses of mirogabalin in healthy Asian volunteers. Clin Pharmacol Drug Dev. 2018;7(6):661-9.

26. Vinik A, Rosenstock J, Sharma U, et al. Efficacy and safety of mirogabalin (DS-5565) for the treatment of diabetic peripheral neuropathic pain: a randomized, double-blind, placebo- and active comparatorcontrolled, adaptive proof-of-concept phase 2 study. Diabetes Care. 2014;37(12):3253-61.

27. Posner K, Brown GK, Stanley B, et al. The ColumbiaSuicide Severity Rating scale: initial validity and internal consistency findings from three multisite studies with adolescents and adults. Am J Psychiatry. 2011;168(12):1266-77.

28. Melzack R. The short-form McGill pain questionnaire. Pain. 1987;30(2):191-7.

29. Guy W. Clinical global impressions (CGI) scale, modified. In: Rush JA, editor. Handbook of psychiatric measures. 1st ed. Washington: American Psychiatric Association; 2000.

30. Finnerup NB, Haroutounian S, Baron R, et al. Neuropathic pain clinical trials: factors associated with decreases in estimated drug efficacy. Pain. 2018;159(11):2339-46.

31. Gilron I. Methodological issues associated with clinical trials in neuropathic pain. Expert Rev Clin Pharmacol. 2016;9(11):1399-402.

32. Tuttle $A H$, Tohyama $S$, Ramsay $\mathrm{T}$, et al. Increasing placebo responses over time in US clinical trials of neuropathic pain. Pain. 2015;156(12):2616-26.

33. Brown K, Mendell J, Ohwada S, et al. Tolerability, pharmacokinetics, and pharmacodynamics of mirogabalin in healthy subjects: results from phase 1 studies. Pharmacol Res Perspect. 2018;6(5): e00418. 
34. Vase L, Skyt I, Hall KT. Placebo, nocebo, and neuropathic pain. Pain. 2016;157(Suppl 1):S98-105.

35. Satoh J, Yagihashi S, Baba M, et al. Efficacy and safety of pregabalin for treating neuropathic pain associated with diabetic peripheral neuropathy: a

14 week, randomized, double-blind, placebo-controlled trial. Diabet Med. 2011;28(1):109-16. 